\title{
IR ENERGY DISTRIBUTION OF OH/IR SOURCES
}

W. A. Sherwood, E. Kreysa and G. V. Schultz

Max-Planck-Institut für Radioastronomle

Auf dem Hügel 69, 5300 Bonn,

Federal Republic of Germany

Summary. Some infrared counterparts of Type II OH/IR sources have been observed between $1 \mu$ and $30_{\mu}$. In spite of their having very similar OH emission spectra, their energy distributions differ considerably among themselves from "flat". (1.25 $\mu$ to $4.9 \mu)$ to "steep" $(2.2 \mu$ to $20 \mu$ ). The energy distributions are compared with more familiar objects.

Defintions of Type II OH/IR sources have been given by H.J. Habing and A. Winnberg within the conference proceedings. The OH spectra of this type are all very similar in shape being characterized by wo emission peaks separated by typically $30 \mathrm{~km} \mathrm{~s}^{-1}$.

However similar they are to each other in their OH emission, they show quite different IR energy distributions. The sources (Schultz and Kreysa, 1976; Schultz et al., 1976) have been observed quasi simultaneously (within one or two months) in OH and IR since 1975 and they do vary together ruling out misidentification (Kreysa et al. , 1978, in preparation). The IR data were obtained in August 1977 at ESO $1 \mathrm{~m}$ telescope using an InSb detector (Kreysa, in preparation) for wavelengths $1.25 \mu$ to $4.9 \mu$ and a Low Ga-Ge bolometer for $10 \mu, 20 \mu$ and $30 \mu$. The data for 24 sources are shown in Fig. 1. The sources have been normalized at $\mathrm{L}[3.6 \mu]$, the wavelength at which we searched for and made the initial identification of the IR counterpart to the OH source. Fig. 1 suggests that selection effects may result from this search procedure (as they certainiy occured in previous searches made at $2.2 \mu(\mathrm{K}))$ : flat spectra $\mathrm{OH} / \mathrm{IR}$ sources may be detected at shorter wavelengths now that better coordinates have been determined and steep spectra sources may be detected only at longer wavelengths, $4.9 \mu$ or $10 \mu$.

The bracketted triangle at $1_{\mu}$ is based on an estimate by J. W. Pel (private communication) of an image tube direct plate of $\mathrm{OH} 20.7+0.1$. If the identification should not prove to be correct then the colour index would be even greater thereby confirming that even the flat IR energy distribution for this object turns down steeply between $1.25 \mu$ and $1 \mu$.

The energy distributions do not change with the IR variations so this camot be the reason for the differences in energy distributions (see Fig. 2 for an example). This confirms an earlier result by Harvey et al. (1974) that the colour index is constant with time.

In Fig. 3 three classes of objects - M stars (Taurids), Carbon Stars (Cygnids) (Stecker and Ney, 1974) and the BN object in Orion (beyond $10 \mu$ it is difficult to separate from the Kleinmann-Low nebula which is very bright) - are compared with the two extremes of the OH /IR sources. From broad band IR photometry it is difficult to say much about the nature 


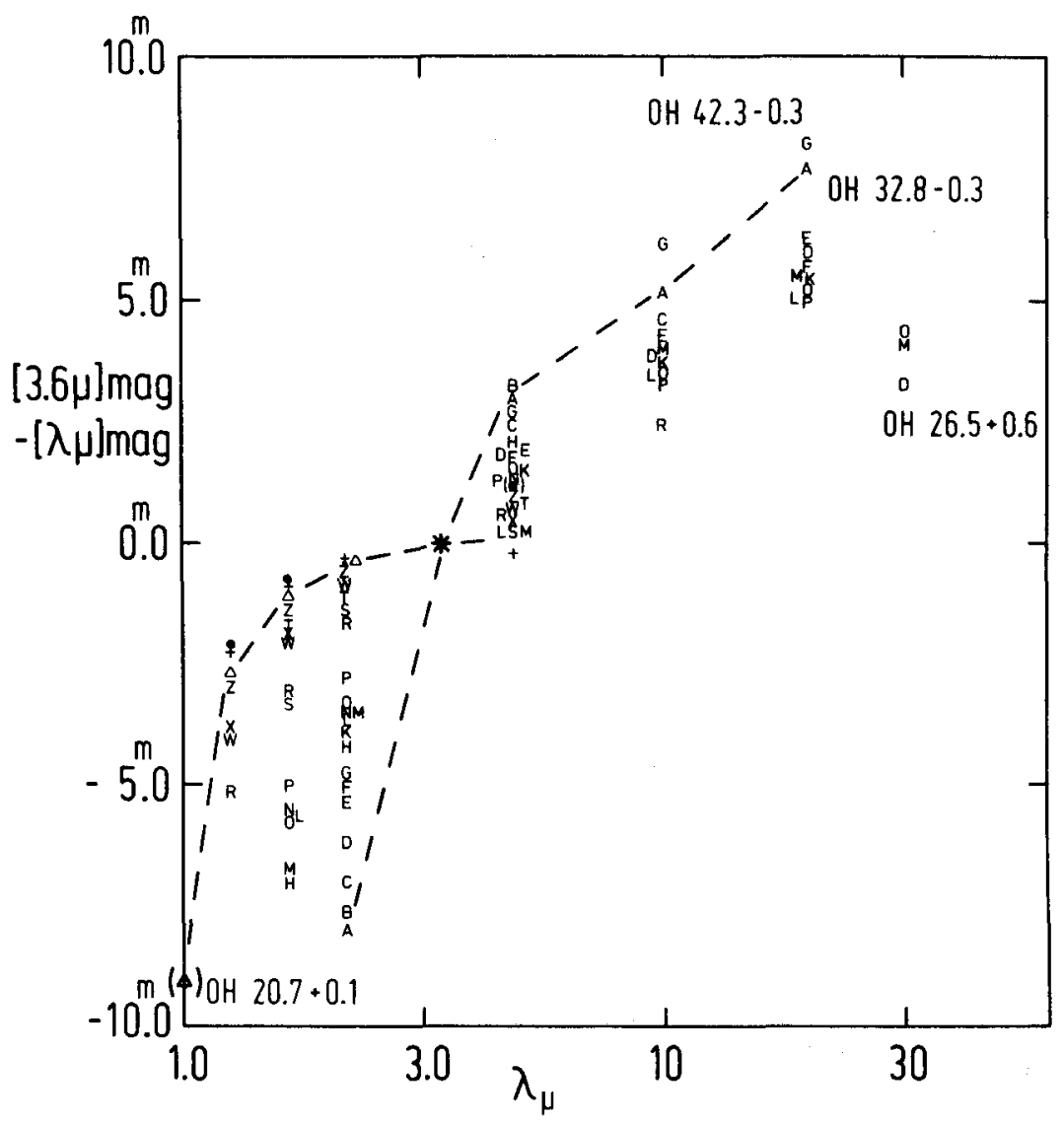

Fig. 1. The broad band infrared energy distributions of $24 \mathrm{OH} / \mathrm{IR}$ sources. Data have been normalized at $L[3.6 \mu]$ and the ordinate is the colour index, $[3.6 \mu] \mathrm{mag}-\lambda \lambda \mu]$ mag, the difference in magnitude between two wavelengths. The sources are represented by letters A... Z, (plus symbols), according to the size of their L-K index. Four sources have been identified: $\mathrm{OH} 26.5+0.6$ (Symbol D) as apparently the strongest source at $1612 \mathrm{MHz}$; OH 32. 8-0.3 (A) has the largest L-K index; OH 42. 3-0.3 (G) has the largest $\mathrm{L}-\mathrm{N}$ index; $\mathrm{OH} 20.7+0.1(\Delta)$ shows an abrupt turnover between 1. $25 \mu$ and $1 \mu$.

of Cygnid, Taurid and $\mathrm{OH} / \mathrm{IR}$ objects. See K. M. Merrill's contribution to this topic within these proceedings. Both $\mathrm{OH} / \mathrm{IR}$ sources are believed to have long period oxygen rich Mira 


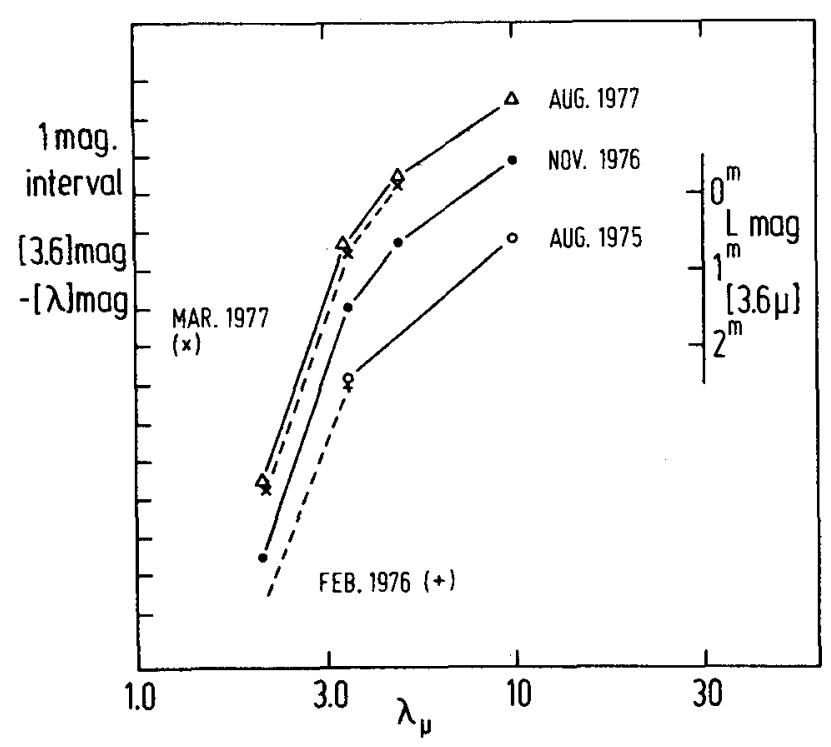

Fig. 2. The relative infrared energy distribution of $\mathrm{OH} 26.5+0.6$ at five different epochs remains the same although the intensity at $L[3.6 \mu]$ as read from the scale inserted at right has varied by nearly two magaitudes. The colour index is given by the left scale.

variables as their central stars while the Cygnids include one non-carbon star, NML Cygni.

Objects having similar $\mathrm{OH}$ spectra produce dissimilar IR objects and the converse is also true; the BN object has an appearance (Russell et al. , 1977) very similar to that of $\mathrm{OH}$ 26. $5+0.6$ (Forrest et al. , 1977) but the BN object does not have any double-peaked 1612 $\mathrm{MHz} \mathrm{OH}$ emission nor does it vary in the infrared.

Bedijn (private communication) suggests that the $10 \mu$ feature differs quite significantly between the $\mathrm{BN}$ object and the $\mathrm{OH} / \mathrm{IR}$ object $26.5+0.6$ and may provide an accurate means of distinguishing them. See also Bedijn et al. , 1977.

We conclude that IR energy distributions of Type II OH/IR sources do not reflect the uniformity of the OH spectra at $1612 \mathrm{MHz}$. It appears that there is no correlation between the amount of IR emission caused by dust and $\mathrm{OH}$ emission, le., that the dust is not responsible for pumping the OH emission. Apparently then, the only thing that all the OH/IR sources have in common is the central Mira star provided the stellar radiation field reaches the $\mathrm{OH}$ molecules. There are several pieces of evidence for this: 


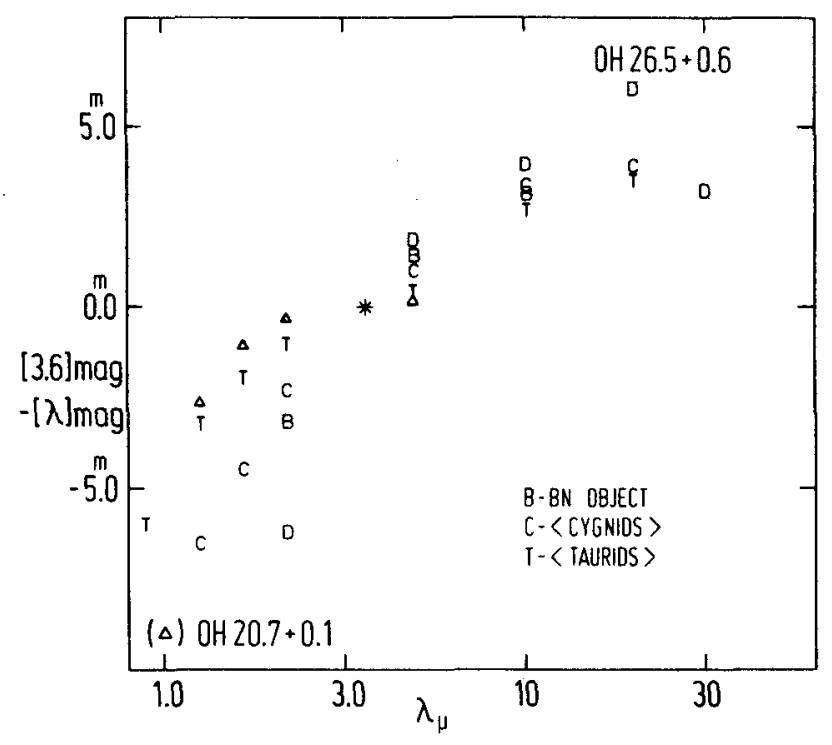

Fig. 3. The mean energy distributions of $M$ stars (Taurids; T) and Carbon stars (Cygnids; C) are compared with the Becklin-Neugebauer (BN; B) object in Orion and two $\mathrm{OH} / \mathrm{IR}$ objects $(\mathrm{OH} 20.7+0.1, \Delta ; \mathrm{OH} 26.5+0.6$, D) taken from Figure 1.

1) Stellar molecular bands are visible in $\mathrm{OH} 26.5+0.6$ at $2.5+\mu$ (Merrill, these proceedings).

2) $\mathrm{OH}$ and Mira vary together when the star is seen (Harvey et al., 1974) ie., when there is little dust.

3) The VLB interferometry shows a very mottled picture of components of $\mathrm{OH}$ emission about the IR source as though the dust clouds prevent $\mathrm{OH}$ from being pumped except where the stellar radiation field leaks through does one find $\mathrm{OH}$ emission.

\section{References}

Bedijn, P.J., Habing, H.J., and de Jong, T. : 1977, NASA TMX 73.190, p. 44

Forrest, W. J., Gillett, F. C., Houck, J. R. , McCarthy, J., Merrill, K. M. , Piper, J. L., Puetter, R. C. , Russell, R.W., Soifer, B. T., Willner, S. P. : 1977, NASA TMX 73. 190, p. 32

Harvey, P. M. , Bechis, K. P. , Wilson, W.J., and Ball, J. A. : 1974, Astrophys. J. Suppl. 27 , No. 248,331

Russell, R. W. , Soifer, B. T. , and Puetter, R. C. : 1977, Astron. Astrophys. 54, 959

Schultz, G. V. , and Kreysa, E. : 1976, Astron. Ges. Mitt. 38, 244

Schultz, G. V., Kreysa, E., and Sherwood, W. A. : 1976, Astron. Astrophys. 50, 171

Stecker, D. W. , and Ney, E. P. : 1974, Astron. J. 79, 797 